\title{
Two Color QCD beyond the BEC régime
}

\author{
Simon Hands ${ }^{*+}$ \\ University of Wales Swansea \\ E-mail: s.hands@swan.ac.uk
}

\section{Seyong Kim}

Sejong University, Seoul

E-mail: skimesejong.ac.kr

\section{Jon-Ivar Skullerud}

Trinity College, Dublin

E-mail: jonivar@maths.tcd.ie

\begin{abstract}
We present results of simulations of Two Color QCD using two flavors of Wilson quark in the fundamental representation, at non-zero quark chemical potential $\mu$, on an $8^{3} \times 16$ lattice. Results for the quark number density, quark and gluon energy densities, and superfluid condensate are qualitatively distinct from the behaviour expected on the assumption that the dominant degrees of freedom are tightly bound scalar diquarks which Bose condense; rather the scaling with $\mu$ is more suggestive of a Fermi surface disrupted by a Cooper pair condensate. We also present evidence both for screening of the static potential, and color deconfinement, arising solely as a result of a non-zero quark density.
\end{abstract}

XXIIIrd International Symposium on Lattice Field Theory

25-30 July 2005

Trinity College, Dublin, Ireland

\footnotetext{
${ }^{*}$ Speaker.

${ }^{\dagger}$ PPARC Senior Research Fellow
} 


\section{Preamble}

QCD with gauge group SU(2) and quark chemical potential $\mu \neq 0$ has enjoyed much recent interest [1]. It lies in a class of models with long-ranged interactions (together with QCD with non-zero isospin chemical potential (ie. $\mu_{u}=-\mu_{d}$ ) and vectorlike theories with adjoint quarks) in which the quark determinant $\operatorname{det} M(\mu)$ is real, and hence amenable to study using standard LGT algorithms.

In Two Color QCD, $q$ and $\bar{q}$ fields live in equivalent representations of the color group SU(2), implying that chiral multiplets contain both $q \bar{q}$ mesons and $q q$ baryons. In the chiral limit where $m_{\pi} \ll m_{\rho}$, where $\rho$ generically denotes any non-Goldstone hadron, it is possible to study the $\mu$ dependence of the model systematically using chiral perturbation theory $(\chi \mathrm{PT})$ [2]. The key result is that the ground state has non-zero quark density $n_{q}>0$ for all $\mu$ greater than some onset value $\mu_{o}=\frac{1}{2} m_{\pi}$. At the same point a superfluid order parameter $\langle q q\rangle \neq 0$ develops, signalling spontaneous breakdown of the original global U(1) baryon number symmetry. Since the transition is second order the matter, consisting of tightly-bound $q q$ scalars, may be arbitrarily dilute, and in the limit $\mu \rightarrow \mu_{o+}$ is a textbook Bose-Einstein Condensate (BEC). In this régime the quantitative predictions of $\chi \mathrm{PT}$ read:

$$
n_{q} \propto f_{\pi}^{2}\left(\mu-\mu_{o}\right) ;\langle q q\rangle \propto \sqrt{1-\left(\frac{\mu_{0}}{\mu}\right)^{4}} \Rightarrow \lim _{\mu \rightarrow \infty}\langle q q(\mu)\rangle=\text { const. }
$$

This behaviour has indeed been confirmed by simulations with staggered fermions $[3,4,5]$

This picture should be contrasted with another paradigm for superfluidity, namely BCS condensation of weakly interacting quark Cooper pairs from opposite points of a Fermi surface with radius $k_{F} \approx E_{F}=\mu$. For such a system, we obtain $n_{q}$ simply by counting states within the Fermi sphere, and the condensate by assuming that only states within a layer of thickness $\Delta \ll \mu$ around the Fermi surface participate in the pairing:

$$
n_{q} \propto \mu^{3} ;\langle q q\rangle \propto \Delta \mu^{2} .
$$

The same rule of thumb predicts the quark energy density $\varepsilon_{q} \propto \mu^{4}$.

We might expect the crossover from BEC to BCS for $\left(\mu-\mu_{o}\right) \gg \frac{1}{2}\left(m_{\rho}-m_{\pi}\right)$, at which point the Goldstones are no longer distinguished hadrons, and the fermionic nature of the constituents comes into play. The chiral limit is thus unimportant, and for this reason we have chosen to revisit Two Color QCD using Wilson fermions [6]. Pioneering simulations by the Hiroshima group appeared in [7]. As shown below, an advantage of the Wilson formulation is that simulations with $N_{f}=2$ quark flavors are possible, ensuring the theory is asymptotically free and confining for $\mu=T=0$ for all couplings, with a controllable continuum limit as $\beta \rightarrow \infty$. This makes the Wilson LGT particularly suitable for a study of gluodynamics in the presence of a background baryon charge density, though it should be stressed that in contrast to 3-color QCD, there is here no physical distinction between fundamental and anti-fundamental charges.

\section{Formulation and Simulation}

The $N_{f}=2$ quark action we want to simulate is as follows:

$$
S=\bar{\psi}_{1} M \psi_{1}+\bar{\psi}_{2} M \psi_{2}-J \bar{\psi}_{1}\left(C \gamma_{5}\right) \tau_{2} \bar{\psi}_{2}^{t r}+\bar{J} \psi_{2}^{t r}\left(C \gamma_{5}\right) \tau_{2} \psi_{1},
$$


where flavor indices are written explicitly, and we have included scalar isoscalar diquark source terms, which will help to reduce IR fluctuations in the superfluid phase [5]. The action coincides with that of [6] with the identification $J=2 \bar{\jmath}, \bar{J}=2 j$. The charge conjugation matrix $C$ defined by $C \gamma_{\mu} C^{-1}=\gamma_{\mu}^{*}$ with $\gamma_{\mu}^{*}=\gamma_{\mu}^{t r}$ in Euclidean metric has the properties $C^{\dagger}=C^{-1}=-C,\left[C, \gamma_{5}\right]=0$. The crucial Two Color identity is $\tau_{2} U_{\mu}(x) \tau_{2}=U_{\mu}^{*}(x)$. The source strengths $J$ and $\bar{J}$ are $a$ priori independent and their signs are arbitrary for now. The matrix $M$ is the textbook Wilson fermion operator

$$
M_{x y}(\mu)=\delta_{x y}-\kappa \sum_{v}\left[\left(1-\gamma_{v}\right) e^{\mu \delta_{v 0}} U_{v}(x) \delta_{y, x+\hat{v}}+\left(1+\gamma_{v}\right) e^{-\mu \delta_{v 0}} U_{v}^{\dagger}(y) \delta_{y, x-\hat{v}}\right]
$$

with properties

$$
\begin{aligned}
\gamma_{5} M(\mu) \gamma_{5} & =M^{\dagger}(-\mu) \\
C \tau_{2} M(\mu) C^{-1} \tau_{2} & =M^{t r}(-\mu) \\
\Rightarrow \quad\left(C \gamma_{5}\right) \tau_{2} M(\mu)\left(C \gamma_{5}\right)^{-1} \tau_{2} & =M^{*}(\mu)
\end{aligned}
$$

Property (2.5) implies $\operatorname{det} M(\mu)$ is real, but because $M$ contains both hermitian and antihermitian non-constant components there is no proof that it is positive. This confirms the impossibility of simulating a single Wilson flavor without a Sign Problem, and also suggests that even for $N_{f}$ even there is an ergodicity problem along the lines of that found for adjoint staggered fermions [4], where changing the sign of $\operatorname{det} M$ requires an eigenvalue to flow through the origin, which the Two-Step Multi-Bosonic algorithm can manage but the Hybrid Monte Carlo (HMC) algorithm not.

Now, with the change of variables $\bar{\phi}=-\psi_{2}^{t r} C \tau_{2}, \phi=C^{-1} \tau_{2} \bar{\psi}_{2}^{t r}$ and the dropping of the index from flavor 1, eq. (2.1) can be recast as:

$$
S=(\bar{\psi}, \bar{\phi})\left(\begin{array}{cc}
M(\mu) & J \gamma_{5} \\
-\bar{J} \gamma_{5} & M(-\mu)
\end{array}\right)\left(\begin{array}{l}
\psi \\
\phi
\end{array}\right) \equiv \bar{\Psi} \mathscr{M} \Psi
$$

Note that the action is bilinear in the variables $\Psi, \bar{\Psi}$, so that the Grassmann integral yields a factor $\operatorname{det} \mathscr{M}$ rather than a Pfaffian. Using the identity

$$
\operatorname{det}\left(\begin{array}{cc}
X & Y \\
W & Z
\end{array}\right)=\operatorname{det} X \operatorname{det}\left(Z-W X^{-1} Y\right)
$$

and properties $(2.3,2.4)$ we deduce

$$
\operatorname{det} \mathscr{M}=\operatorname{det}\left(M^{\dagger}(\mu) M(\mu)+J \bar{J}\right)
$$

Hence positivity of $\operatorname{det} \mathscr{M}$ requires the product $J \bar{J}$ to be real and positive, which translates into the requirement that the diquark source term be antihermitian [6]. Since no eigenvalue of $\mathscr{M}$ can vanish, the ergodicity problem is also cured.

Now use (2.6) to write

$$
\mathscr{M}^{\dagger} \mathscr{M}=\left(\begin{array}{ll}
M^{\dagger}(\mu) M(\mu)+\mid J^{2} & M^{\dagger}(-\mu) M(-\mu)+|J|^{2}
\end{array}\right) .
$$


The off-diagonal terms can be shown to vanish if $\bar{J}=J^{*}$ using (2.3); moreover the same identity applied to the lower block yields

$$
\operatorname{det} \mathscr{M}^{\dagger} \mathscr{M}=\left[\operatorname{det}\left(M^{\dagger}(\mu) M(\mu)+\bar{J} J\right)\right]^{2} .
$$

It is therefore possible to take the square root analytically, by using pseudofermion fields with weight $\left(M^{\dagger} M+|J|^{2}\right)^{-1}$. This has the advantage of $(a)$ requiring matrix/vector multiplications of the half the dimensionality, and $(b)$ permitting a hamiltonian evaluation and hence the use of an exact HMC algorithm. It is equivalent to the even/odd partitioning step used for staggered fermion gauge theories, but is more transparent since all lattice sites are physically equivalent, making the force term easier to implement. The trick was used in [5], though because the staggered version still requires a Pfaffian rather than a determinant, an HMD algorithm was used in that case.

\section{Observables}

The most fundamental is the quark density, obtained as a derivative of the free energy:

$$
\begin{aligned}
n_{q} \equiv-\frac{1}{V} \frac{\partial \ln \mathscr{Z}}{\partial \mu}= & \frac{1}{V \mathscr{Z}} \int D \Psi D \bar{\Psi}(\bar{\psi}, \bar{\phi})\left(\begin{array}{l}
\frac{\partial M}{\partial \mu} \\
-\frac{\partial M}{\partial \mu}
\end{array}\right)\left(\begin{array}{l}
\psi \\
\phi
\end{array}\right) e^{-S} \\
= & \kappa\left\langle\bar{\psi}_{x}\left(\gamma_{0}-1\right) e^{\mu} U_{0}(x) \psi_{x+\hat{0}}+\bar{\psi}_{x}\left(\gamma_{0}+1\right) e^{-\mu} U_{0}^{\dagger}(x-\hat{0}) \psi_{x-\hat{0}}\right\rangle \\
& -\kappa\left\langle\bar{\phi}_{x}\left(\gamma_{0}-1\right) e^{\mu} U_{0}(x) \phi_{x+\hat{0}}+\bar{\phi}_{x}\left(\gamma_{0}+1\right) e^{-\mu} U_{0}^{\dagger}(x-\hat{0}) \phi_{x-\hat{0}}\right\rangle .
\end{aligned}
$$

The apparently unphysical term $-2 \kappa\left\langle\bar{\psi}\left(\partial_{0}-\mu\right) \psi\right\rangle$ is irrelevant in the long wavelength limit. We have checked that at saturation $\lim _{\mu \rightarrow \infty} n_{q}=2 N_{f} N_{c}$. The quark energy density $\varepsilon_{q}$ is approximated by the same expression with a relative minus sign between forward and backward timelike links.

For the diquark condensate, it is convenient first to introduce rescaled source strengths $\{j, \bar{j}\}=$ $\kappa^{-1}\{J, \bar{J}\}$, and then the orthogonal combinations $j_{ \pm}=j \pm \bar{\jmath}$. We then have

$$
\left\langle q q_{ \pm}\right\rangle \equiv-\frac{1}{V} \frac{\partial \ln \mathscr{Z}}{\partial j_{ \pm}}=\frac{\kappa}{2}\left\langle\bar{\psi} \gamma_{5} \phi \mp \bar{\phi} \gamma_{5} \psi\right\rangle
$$

Since the diquark condensate is not a component of a conserved current, its normalisation is to some extent arbitrary: we prefer to normalise it with the same factor of $\kappa$ as the quark density.

\section{Results}

We have studied an $8^{3} \times 16$ lattice with parameters $\beta=1.7, \kappa=0.1780$, using a standard Wilson plaquette action. Unfortunately our results are not directly comparable with those of [7] due to their use of an improved gauge action. Studies of the $\mu=0$ string tension yield a lattice spacing $a=0.220 \mathrm{fm}$, and the spectrum $m_{\pi} a=0.800(2), m_{\pi} / m_{\rho}=0.920(3)$ [6]. In simulations with $\mu \neq 0$ we have used a diquark source $j a=0.04$. So far we have accumulated roughly 300 HMC trajectories of mean length 0.5 for $\mu a \in[0.3,0.9]$.

In Fig. 1 we plot quark observables (with a suitable subtraction so that $\varepsilon_{q}(\mu)=0$ ), and in Fig. 2 the gluon energy density $\varepsilon_{g}=\frac{3 \beta}{2}\left\langle\operatorname{tr}\left(\square_{t}-\square_{s}\right)\right\rangle$ and Polyakov line, by far the noisiest observable. The behaviour of the superfluid condensate $\langle q q\rangle$ vs. $\mu$ is qualitatively very different from the 


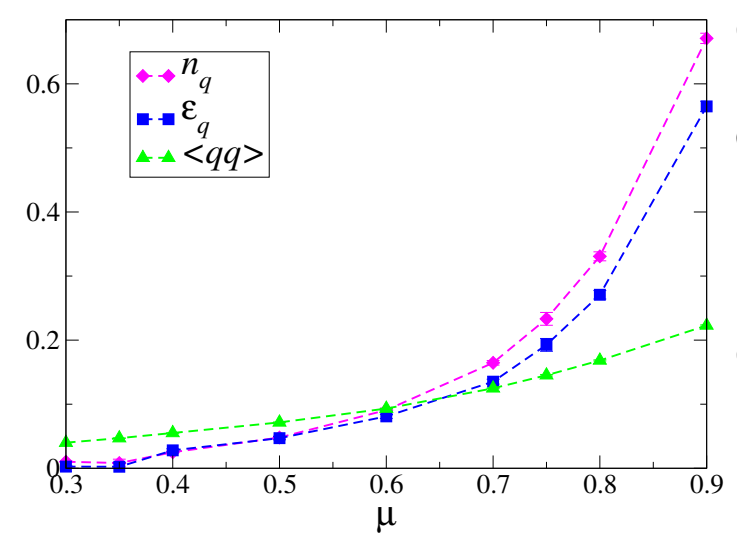

Figure 1: Quark observables

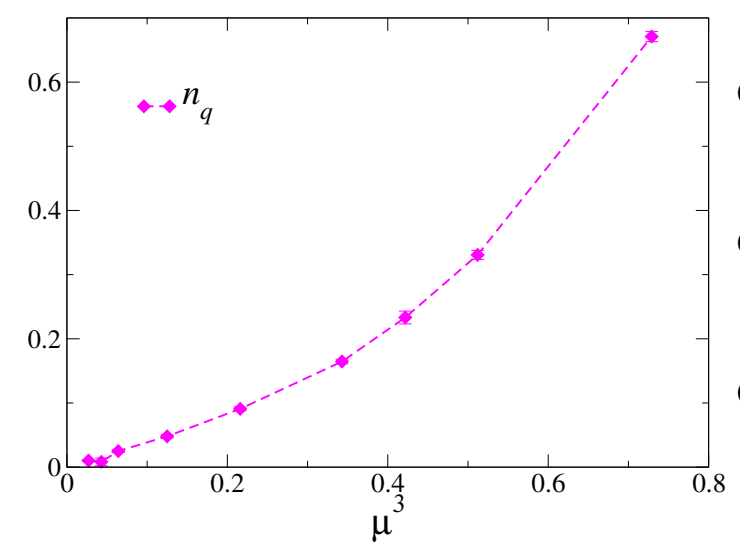

Figure 3: Quark number density vs. $\mu^{3}$

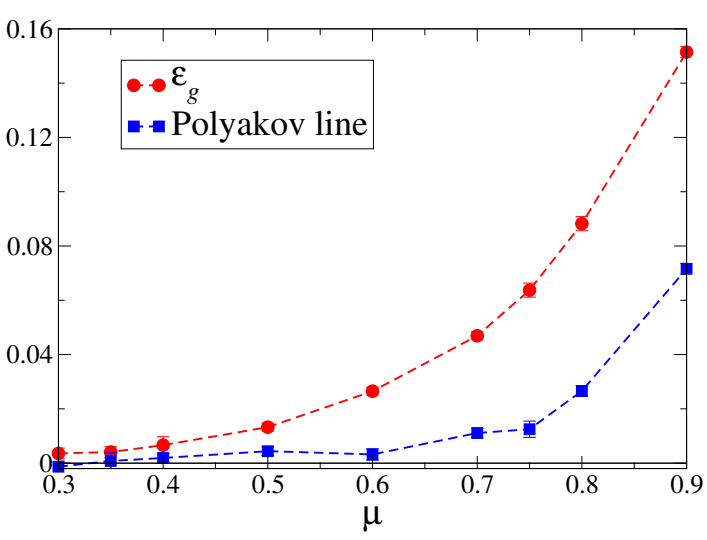

Figure 2: Gluon observables

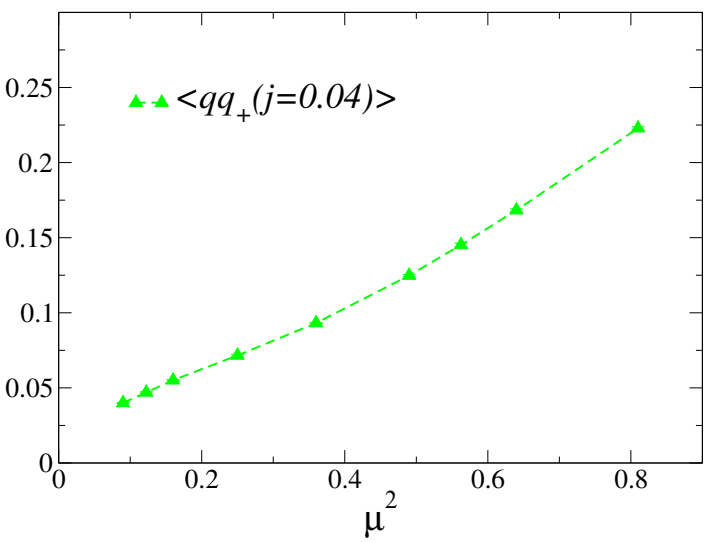

Figure 4: Diquark condensate vs. $\mu^{2}$

negative curvature seen in the $j \rightarrow 0$ limit in studies with staggered fermions[3, 4, 5]. Moreover the magnitude of $n_{q}(\mu)$ is much smaller, suggesting following (1.1) that the effective $f_{\pi}$ is much smaller, and hence the hadron degrees of freedom more strongly interacting.

This motivates us to replot the data following (1.2), as shown in Figs. $3 \& 4$. There is some evidence to support the formation of a Fermi sphere; $n_{q}$ increases if anything more rapidly than $\mu^{3}$, although the impact of lattice artifacts has still to be determined. Similarly, the condensate is consistent with BCS behaviour being recovered in the $j \rightarrow 0$ limit, though data at smaller $j$ will be required to confirm this. The most spectacular evidence for a Fermi sphere comes from the energy densities, plotted in Fig. 5. A significant fraction of the energy density, $O(30 \%)$, comes from gluons, suggesting that the medium which forms is strongly-interacting. Finally in Fig. 6 we show the static quark potential $V(r)$, showing clear evidence for screening for $\mu a \geq 0.4$.

\section{Outlook}

The parameter set chosen for our initial study appear to place the Two Color quark medium in a qualitatively different régime from that described by $\chi \mathrm{PT}$ and studied numerically using staggered fermions. If our guess that the system has a Fermi surface disrupted by a diquark condensate is borne out by further analysis, then the system may have a lot more in common with high density 


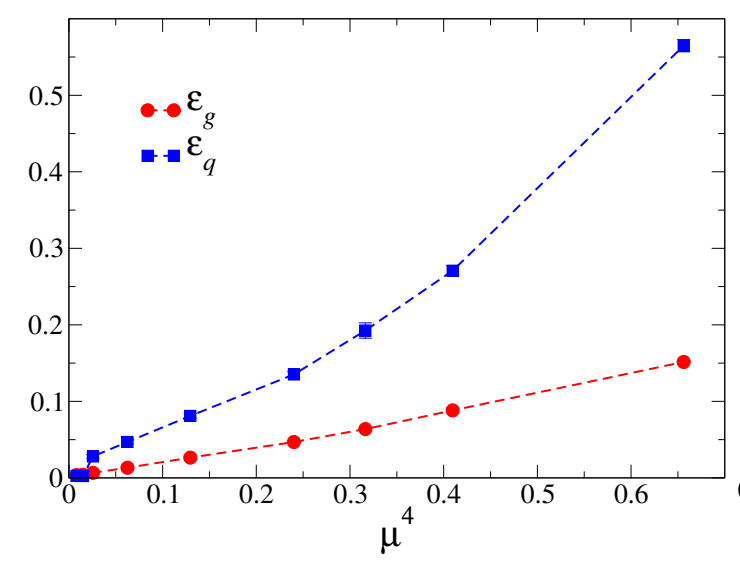

Figure 5: Quark and gluon energy densities vs. $\mu^{4}$

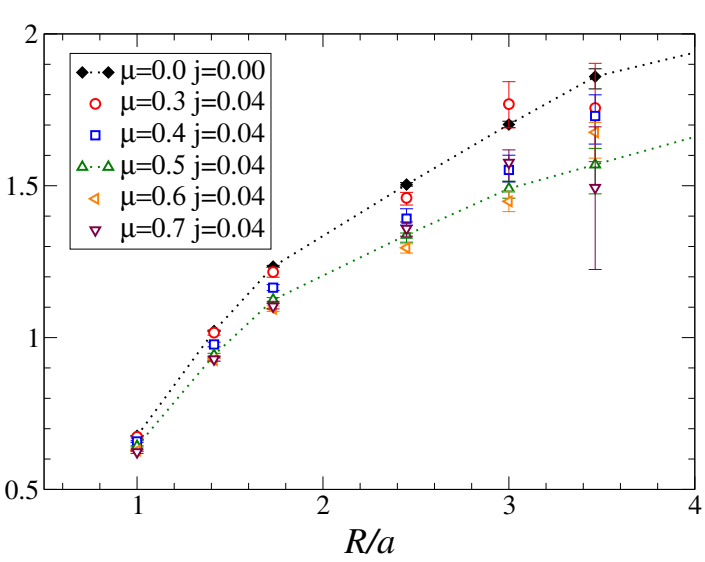

Figure 6: Static potential $V(r)$ for various $\mu$

QCD than originally thought. One exciting possibility is that the onset transition predicted by $\chi \mathrm{PT}$ at $\mu_{o} a \simeq 0.4$ does not coincide with the deconfining transition, which the Polyakov loop data of Fig. 2 suggest may not occur until $\mu a \approx 0.6(1)$. Future study will also focus on the hadron spectrum, inspired by the evidence for decrease in mass for vector states reported in [7], and a study of the gluon propagator.

\section{Acknowledgements}

SK thanks PPARC for support during his visit to Swansea in 2004/05. We have also benefitted from discussions with Kim Splittorff, and wish to warmly thank Shinji Ejiri and Luigi Scorzato for their participation in the early stages of this project.

\section{References}

[1] S.J. Hands, Lattice Matter, Nucl. Phys. Proc. Suppl. 106 (2002) 142 [hep-lat/ 0109034 ].

[2] J.B. Kogut, M.A. Stephanov, D. Toublan, J.J.M. Verbaarschot and A. Zhitnitsky, QCD-like theories at finite baryon density, Nucl. Phys. B 582 (2000) 477 [hep-ph/ 0001171$].$

[3] R. Aloisio, V. Azcoiti, G. Di Carlo, A. Galante and A.F. Grillo, Fermion condensates in finite density QCD at strong coupling, Phys. Lett. B 493 (2000) 189 [hep-lat / 0009034 ].

[4] S.J. Hands, I. Montvay, S.E. Morrison, M. Oevers, L. Scorzato and J.I. Skullerud, Numerical study of dense adjoint matter in two Color QCD, Eur. Phys. J. C 17 (2000) 285 [hep-lat/ 0006018 ];

S.J. Hands, I. Montvay, L. Scorzato and J.I. Skullerud, Diquark condensation in dense adjoint matter, Eur. Phys. J. C 22 (2001) 451 [hep-lat/ 0109029 ].

[5] J.B. Kogut, D.K. Sinclair, S.J. Hands and S.E. Morrison, Two Color QCD at nonzero quark number density, Phys. Rev. D 64 (2001) 094505 [hep-lat/ 0105026 ].

[6] J.I. Skullerud, S. Ejiri, S.J. Hands and L. Scorzato, Lattice simulations of Two Color QCD with Wilson fermions, Prog. Theor. Phys. Suppl. 153 (2004) 60 [hep-lat/ 03120 02].

[7] S. Muroya, A. Nakamura and C. Nonaka, Behavior of hadrons at finite density: lattice study of color SU(2) QCD, Phys. Lett. B 551 (2003) 305 [hep-lat/ 0211010 ]. 